WS15-C02

\title{
Distributed Acoustic Sensing (DAS) for Reservoir Monitoring with VSP
}

A. Mateeva* (Shell), J. Lopez (Shell), R. Detomo (Shell), H. Potters (Shell), W. Berlang (Shell), S. Grandi (Shell), J. Mestayer (Shell), P. Wills (Shell), B. Cox (Shell) \& D. Kiyashchenko (Shell)

\section{SUMMARY}

3D VSP has long been viewed as conceptually attractive for illuminating targets under complex overburden. However, the wide-spread use of 3D VSP has been hindered by the cost and risk of deploying geophones in a borehole, and by the limited availability of accessible wells. These hurdles are largely removed when acquiring downhole seismic with Distributed Acoustic Sensing (DAS) instead of geophones.

Over the past several years Shell has acquired more than 20 DAS VSP-s around the world to test DAS performance and further its development. Early on we established the ability of DAS to provide fit-forpurpose VSP products such as checkshots, images, and time-lapse images in a variety of field situations. More recently, our focus has been on identifying VSP applications through which DAS can add most value. High on our list is reservoir monitoring for fields undergoing stimulation, such as water injection or Thermal EOR, particularly under complex overburden.

In this talk we will show how DAS enables reservoir monitoring through VSP techniques that were unfeasible or cost-prohibitive with geophones. Those include frequent time-lapse monitoring onshore, in deepwater, in environmentally sensitive or congested areas, and simultaneously in many wells for fullfield coverage with 3D VSP. 


\section{Amsterdam ' 14}

3D VSP has long been viewed as conceptually attractive for illuminating targets under complex overburden. However, the wide-spread use of 3D VSP has been hindered by the cost and risk of deploying geophones in a borehole, and by the limited availability of accessible wells. These hurdles are largely removed when acquiring downhole seismic with Distributed Acoustic Sensing (DAS) instead of geophones.

Over the past several years Shell has acquired more than 20 DAS VSP-s around the world to test DAS performance and further its development. Early on we established the ability of DAS to provide fitfor-purpose VSP products such as checkshots, images, and time-lapse images in a variety of field situations. More recently, our focus has been on identifying VSP applications through which DAS can add most value. High on our list is reservoir monitoring for fields undergoing stimulation, such as water injection or Thermal EOR, particularly under complex overburden.

In this talk we will show how DAS enables reservoir monitoring through VSP techniques that were unfeasible or cost-prohibitive with geophones. Those include frequent time-lapse monitoring onshore, in deepwater, in environmentally sensitive or congested areas, and simultaneously in many wells for full-field coverage with 3D VSP. 